\title{
Iris recognition by using blood vessel segmentation
}

\author{
Madhuri W. Patil ${ }^{2}$, Mr. C S Patil ${ }^{2}$, Mr. Rajesh R Karhe ${ }^{3}$ \\ ${ }^{I}$ Depart of Electronics and Commmunication, Shri Gulabrao Deokar College of Engineering, Jalgaon. \\ ${ }^{2}$ Head of the Department Depart of Electronics and Commmunication, Shri Gulabrao Deokar College of \\ Engineering, Jalgaon. \\ ${ }^{3}$ Associate Professor Depart of Electronics and Commmunication, Shri Gulabrao Deokar College of \\ Engineering, Jalgaon.
}

\begin{abstract}
Among the various biometrics technologies, iris recognition is the most reliable and accurate biometric identification system available. In this paper a novel framework for recognizing and identifying iris is been proposed. Iroimages are pre-processed to remove noise using median filtering on different image planes separately. GLCM and Gabor wavelet texture features have been used to identify the Iris and classification tree approach is used to classify them. The results are improved by the use of combined GLCM and Gabor features. Speed vector machine (SVM) is used for classification. This is an intelligent system which has the ability to identify retina from photographs of their iris and it provides accurate results in less time. The real time database is prepared for the experimental use. The database contains various iris with various shapes, colours and size. Experiment is carried out with the different Iris of different classes and tested.
\end{abstract}

\section{Introduction}

The need for infallible security systems has become a vital aspect in public security. Iris recognition is one of the important techniques and compared with other biometric features (such as face, voice, etc.), the iris is more stable and reliable for identification. Iris recognition is the analysis of the coloured ring that surrounds the pupil. The iris has unique structure and these patterns are randomly distributed, which can be used for identification of human being.

Retina: In the center of the retina is the optic nerve, a circular to oval white area measuring about $2 \times 1.5$ $\mathrm{mm}$ across. From the center of the optic nerve radiates the major blood vessels of the retina.

Approximately 17 degrees (4.5-5 mm), or two and half disc diameters to the left of the disc, can be seen the slightly oval-shaped, blood vessel-free reddish spot, the fovea, which is at the center of the area known as the macula by ophthalmologists Unlike traditionally used biometric systems, vascular pattern of human retina is the most reliable and stable source for biometrics. It is not easy to forge it as it lies at

back end of human eye and is not directly accessible [2]. There is always a confusion regarding iris and retina as they both belong to human eye but their functions and patterns are completely different. Iris is the colored region between the pupil and sclera whereas retina is located at back region of eye. The foundation of retinal recognition is the pattern of blood vessels present in human retina [2]. Figure 1 shows the human retinal and vascular pattern extracted from digital retinal image.

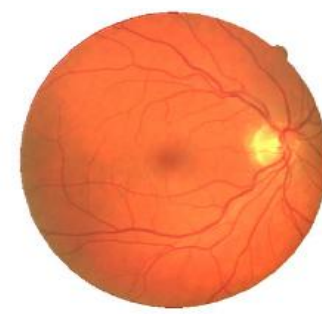

(a)

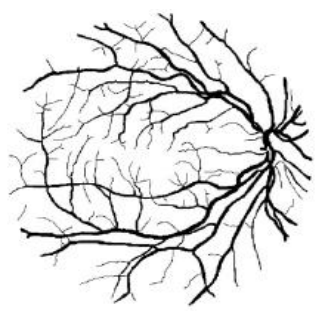

(b)

Figure 1 a. Digital Image of Human Retina $\quad$ b. Vascular Pattern

In today's networked world, the need to maintain the security of information or physical property is becoming both increasingly important and difficult. Most of the times, criminals have been taking advantage of a fundamental flaw in the conventional access control systems. The access control systems based on biometrics have a potential to overcome most of the deficiencies of current security systems and have been gaining importance in recent years. 


\section{Related work for noise removal}

Image noise is the random variation of brightness or color information in images produced by the sensor and circuitry of a scanner or digital camera. Image noise can also originate in film grain and in the unavoidable shot noise of an ideal photon detector [3].Image noise is generally regarded as an undesirable byproduct of image capture. Although these unwanted fluctuations became known as "noise" by analogy with unwanted sound they are inaudible and such as dithering. The types of Noise are Amplifier noise (Gaussian noise), Salt-and-pepper noise, Shot noise (Poisson noise), Speckle noise.

Image de-noising is very important task in image processing for the analysis of images. Ample image de-noising algorithms are available, but the best one should remove the noise completely from the image, while preserving the details. Broadly speaking, De-noising filters can be classified in the following categories:

\section{A.Mean Filter}

Mean filter is an averaging linear filter. Here the filter computes the average value of the corrupted image in a predefined area. Then the center pixel intensity value is replaced by that average value. This process is repeated for all pixel values in the image.

The algorithm proposed in [4] considers first order neighborhood pixels for detecting the noisy pixel and mean filter is considered. Color images are also de-noised by extracting the $\mathrm{R}, \mathrm{G}$ and $\mathrm{B}$ pixels from noisy image and then they are de-noised separately and then merged together to again form the color image. The algorithm is compared with all other standard and well known algorithms and found to have good noise removal capabilities at high densities.

In order to improve the speed of mean filter algorithm and to reduce the redundancy operation, an efficient algorithm is presented in [5]. At first, an assistant array is built up to store the pixels' value summation of each column. Then, according to the relationship between adjacent windows when filter window is siding along the rows, a recursive formula for calculating the midpoint mean of a new window is schemed out. Meanwhile, the recursive formula for updating assistant array items is obtained based on the relationship between adjacent rows' pixels in the changing row process of midpoint of window. On the basis of these two recursion formulas, a high efficient mean filter algorithm is achieved.

\section{B. Median Filter}

The Median filter is a nonlinear digital filtering technique, often used to remove noise. Such noise reduction is a typical preprocessing step to improve the results of later processing (for example, edge detection on an image). Median filtering is very widely used in digital image processing because under certain conditions, it preserves edges whilst removing noise. The main idea of the median filter is to run through the signal entry by entry, replacing each entry with the median of neighboring entries. Note that if the window has an odd number of entries, then the median is simple to define: it is just the middle value after all the entries in the window are sorted numerically. For an even number of entries, there is more than one possible median. The median filter is a robust filter. Median filters are widely used as smoothers for image processing, as well as in signal processing and time series processing. A major advantage of the median filter over linear filters is that the median filter can eliminate the effect of input noise values with extremely large magnitudes.

Authors in [6] proposes a novel decision-based median filter that replaces each corrupted pixel with the median value of their $k$-nearest noise-free pixels. Advantages of the median filter using $k$-nearest noise-free pixels instead of $k$-nearest pixels are two facets: first, it guarantees that pixels after being restored must be noisefree, because the median filter operator is executed on noise-free pixels; second, the median filter using $k$-nearest noise-free pixels adaptively adjusts its window size for each pixel such that the number of noise-free pixels locating in the window increases up to $k$. To realize it, the median filter using $k$-nearest noise-free pixels firstly detects noise-free pixels in an image, then replaces each corrupted pixel with the median value of their $k$ nearest noise-free pixels.

[7] Presents the removal of high density salt and pepper noise in gray scale Images using fuzzy based median filter (FBMF) algorithm. FBMF replaces the noisy pixel by median value when O's, 255's and other pixel values are present in the chosen window and when all pixel values are either O's or 255's, or combination of these, then the noise pixel is replaced by fuzzy membership value of a selected window.

\section{Wiener Filter}

The goal of the Wiener filter is to filter out noise that has corrupted a signal. It is based on a statistical approach. Typical filters are designed for a desired frequency response. The Wiener filter approaches filtering from a different angle. One is assumed to have knowledge of the spectral properties of the original signal and the noise, and one seeks the LTI filter whose output would come as close to the original signal as possible. 
A new widely linear noise-reduction Wiener filter based on the variance and pseudo-variance of the short-time Fourier transform coefficients of speech signals is proposed in [8]. Authors show that this new noise-reduction filter has many interesting properties, including but not limited to: 1) it causes less speech distortion as compared to the classical noise-reduction Wiener filter; 2) its minimum mean squared error (MSE) is smaller than that of the classical Wiener filter; 3) it can increase the subband signal-to-noise ratio (SNR), while the classical Wiener filter has no effect on the subband SNR for any given signal frame and subband.

An adaptive wiener filter design method is presented in [9] using quality based hybrid algorithms. The proposed method restore degraded image using Wiener filter with Gradient based smoothing and Median filter with an adaption of Bilateral filter for edge preserving and Guided filter for reducing staircase effect and gradient reversal. Using this method, clear images are obtained from degraded images without increasing noise, ringing artifacts and halo effect.

\section{Wavelet Transform}

In several applications, it might be essential to analyze a given signal. The structure and features of the given signal may be better understood by transforming the data into another domain. There are several transforms available like the Fourier transform, Hilbert transform, wavelet transform, etc. The Fourier transform is probably the most popular transform. However the Fourier transform gives only the frequencyamplitude representation of the raw signal. The time information is lost. So we cannot use the Fourier transform in applications which require both time as well as frequency information at the same time. The Short Time Fourier Transform (STFT) was developed to overcome this drawback.

According to the characteristic of river sediment image, the two-dimensional image data is transformed into one-dimensional array data from horizontal, vertical, + diagonal line, - the diagonal line four directions, by using different threshold value on the different wavelet decomposition high frequency coefficient and enhancing the processed coefficient, de-noise image is obtained after inversing wavelet transform and calculating on four two-dimensional pictures by unequal coefficient. The results indicate the proposed algorithm in [10] is effective both in reserving the edge and in removing noise.

Authors in [11] have proposed an improved wavelets shrinkage method that uses different parameters across different wavelet scales to denoise the Besov images and it outperforms the existing á fixed algorithms. It is obvious that different parts of an image or different images have different structures and properties, so we assume that these differences could be described by means of their distinct smoothness properties. In experiments, authors used three simple mathematic functions to denote the relationship between the smoothness index and wavelet scale and found that the linear function achieved a better denoising effect.

\section{A. Order Statistics Filter}

Order-Statistics filters are non-linear filters whose response depends on the ordering of pixels encompassed by the filter area. When the center value of the pixel in the image area is replaced by 100 th percentile, the filter is called max-filter. On the other hand, if the same pixel value is replaced by 0 th percentile, the filter is termed as minimum filter.

Directional order statistics filtering approaches are performance limited owing to their implementation with larger window sizes (to a maximum size of $9 \mathrm{x} 9$ ) and with the need of iterative operation to filter the residual noise for highly corrupted images. Authors in [12] presents a methodology to improve the performance of directional order statistics filters for suppression of salt-and-pepper noise. The proposed filtering approach involves eight directions to identify boundary and no boundary pixels. If the non-boundary pixels are noise free, they are left unprocessed otherwise; updated by order statistics parameters of the local window.

A theoretical study and experimental validation on a binaural hearing aid setup of this standard SDWMWF implementation is presented in [13], where the effect of estimation errors in the second-order statistics is analyzed. In this case, and for a single target speech source, the standard SDW-MWF implementation is found not to behave as predicted theoretically. Second, two recently introduced alternative filters, namely the rank-one SDW-MWF and the spatial prediction SDW-MWF, are also studied in the presence of estimation errors in the second-order statistics. These filters implicitly assume a single target speech source, but still only rely on the speech and noise correlation matrices. It is proven theoretically and illustrated through experiments that these alternative SDW-MWF implementations behave close to the theoretical optimum, and hence outperform the standard SDW-MWF implementation.

\section{Feature extraction related work}

Li et. al., introduced a new method to detect vascular bifurcations and crossovers in fundus images. The Gaussian filter is applied to the blue channel of the original color retinal images to suppress the central reflex and reduce the candidate points. The eigenvalues and eigenvectors of Hessian matrix are then obtained in 
multiple scales to provide the structural and directional information. By computing the anisotropy and isotropy of neighboring image segments for each pixel in a retinal image, a multi-scale vessel filter is defined which combines the responses of tubular structures and the responses of bifurcations and crossovers [32].

Azzopardi and Petco, proposed a method for automatic detection of vascular bifurcations in segmented retinal images using trainable COSFIRE filters. The vascular tree observed in a retinal fundus image can provide clues for cardiovascular diseases. Its analysis requires the identification of vessel bifurcations and crossovers. Authors use a set of trainable key point detectors that are called as Combination of Shifted Filter Responses or COSFIRE filters to automatically detect vascular bifurcations in segmented retinal images [33].

Bhuiyan et. al., introduced a method to detect and classify the vascular bifurcation, branch and crossover points (landmarks) based on the vessel geometrical features. They utilize the vessel's centerline and width information to detect and classify these landmarks, which can be used for image matching in medical diagnosis and biometric security applications. The geometrical properties of the blood vessels passing through the potential landmarks are obtained. Perceptual grouping and Support Vector Machine (SVM) are used to classify the landmarks into the vascular bifurcations, branches and crossovers [34].

Ardizzone et. al., presents an effective algorithm for automated extraction of the vascular tree in retinal images, including bifurcations, crossovers and endpoints detection. Correct identification of these features in the ocular fundus helps the diagnosis of important systematic diseases, such as diabetes and hypertension. The pre-processing consists in artifacts removal based on anisotropic diffusion filter. Then a matched filter is applied to enhance blood vessels. The filter uses a full adaptive kernel because each vessel has a proper orientation and thickness. The kernel of the filter needs to be rotated for all possible directions. As a consequence, a suitable kernel has been designed to match this requirement. The maximum filter response is retained for each pixel and the contrast is increased again to make easier the next step. A threshold operator is applied to obtain a binary image of the vascular tree [35].

\section{Development Of The Proposed System}

Development of system include Pattern recognition which is a very important field within computer vision, and the aim of pattern recognition/classification is to classify or recognize the patterns based on extracted features from them. The pattern recognition involves three steps (1) Pre-processing (2) blood vessel segmentation (3) Feature Extraction (4) Classification. In Pre-processing one usually process the image data so it should be in suitable form e.g. one gets an isolated objects after this step. In second step measure the properties of object of interest and in third step, determine the class of object based on features. A brief explanation on the pattern recognition is given in the Figure 1.

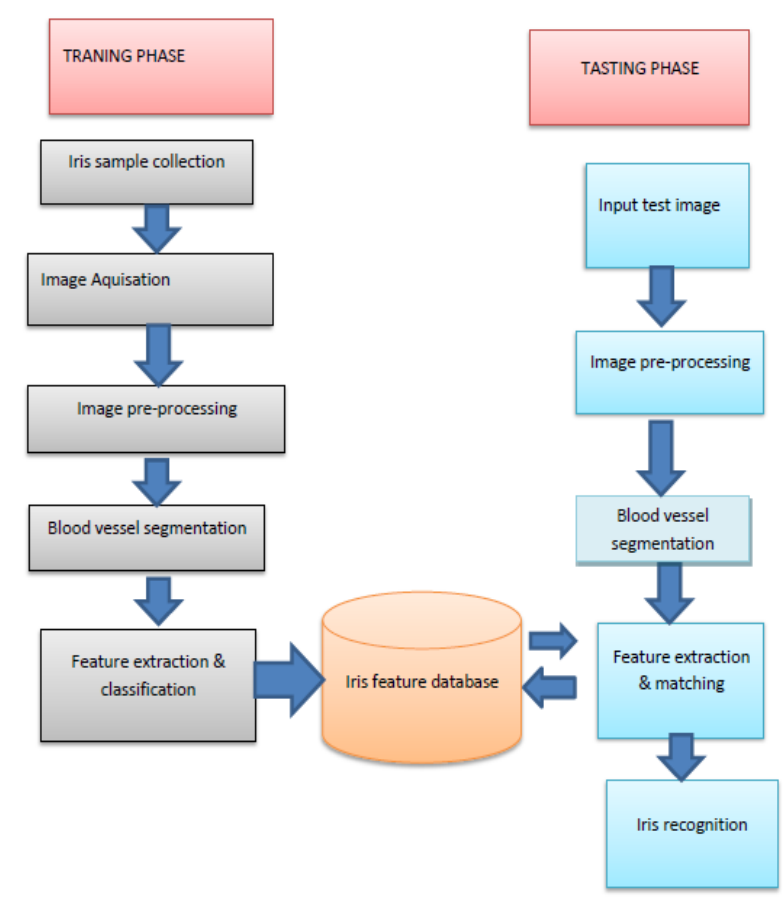

1. Main steps of iris recognition

These steps can be further broken down into sub-steps. 


\section{Training:}

1. Pre-processing: Process the data so it is in a suitable form. Here we have used median filter to remove noise from the iris images.

2. Blood vessel segmentation: blood vessel segmentation takes place by using morphological feature

3. Feature extraction: Extract the features from the training iris images such as GLCM and wavelet features.

4. Dimensionality Reduction: Apply Support vector machine (SVM) on the extracted features. Reduce the amount of data by extracting relevant information.

5. Model Estimation: From the finite set of feature vectors, need to estimate a model (usually statistical) for each class of the training data. Here we have constructed the decision tree model for IRIS recognition

\section{Recognition:}

1. Pre-processing: Process the test data so it is in a suitable form. Here we have used median filter to remove noise from the iris images.

2. Blood vessel segmentation: blood vessel segmentation takes place by using morphological feature.

3. Feature extraction: Extract the features from the test Iris images such as GLCM and wavelet features.

4. Dimensionality Reduction: Apply Support vector machine (SVM) on the extracted features. Reduce the amount of data by extracting relevant information.

5. Classification: Compare feature vectors to the various models and find the closest match. One can match the feature vectors obtained in training set. Here we have used the constructed decision tree model for prediction of the iris class.

\section{A. Image Pre-processing}

Digital images are prone to a variety of types of noise. Noise is the result of errors in the image acquisition process that result in pixel values that do not reflect the true intensities of the real scene. There are several ways that noise can be introduced into an image, depending on how the image is created. The median filter is used to reduce noise in an image, somewhat like the mean filter.

\section{B.Blood Vessel Segmentation: \\ Algorithm:}

1. Read Image.

2. Resize image for easier computation.

3. Convert RGB to Gray .

4. Contrast Enhancement of gray image using adaptive histogram equalization.

5. Background Exclusion by using Average Filter.

6. Take the difference between the gray image and Average Filter Image.

7. Threshold the image using the IsoData Method.

8. Convert to Binary

9. Remove small pixels by using morphological open operation

10. Overlay the images

\section{Feature Extraction}

1. Texture Analysis Using Gabor function and wavelets. We employed Gabor filters to extract textures of different sizes and orientations (i.e. Gabor-based texture feature

2.Texture Analysis Using the Gray-Level Co-Occurrence Matrix (GLCM)

A statistical method of examining texture that considers the spatial relationship of pixels is the gray-level cooccurrence matrix (GLCM), also known as the gray-level spatial dependence matrix. The GLCM functions characterize the texture of an image by calculating how often pairs of pixel with specific values and in a specified spatial relationship occur in an image, creating a GLCM, and then extracting statistical measures from matrix. (The texture filter functions, described in Texture Analysis cannot provide information about shape, i.e., the spatial relationships of pixels in an image.)

\section{Classification (Recognition) by using SVM:}

Once the features have been extracted, then these features are to be used to classify and identify the Iris using SVM classifier to classify Iris based on texture-related features of iris such as GLCM features like contrast, correlation, energy and homogeneity and wavelet features like Gabor. 


\section{Performance Analysis}

System performance, comparisons against the state-of-the art, as well as quantitative and qualitative analysis of how different system configurations and parameters affect performance.

\section{A. Drive Database}

The DRIVE database has been established to enable comparative studies on segmentation of blood vessels in retinal images. The research community is invited to test their algorithms on this database and share the results with other researchers through this web site. On this page, instructions can be found on downloading the database and uploading results, and the results of various methods can be inspected.

\section{B. Result Analysis}

GUI of the system:

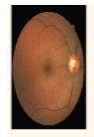

Iris Recognition Using Blood Vessel Segmentation

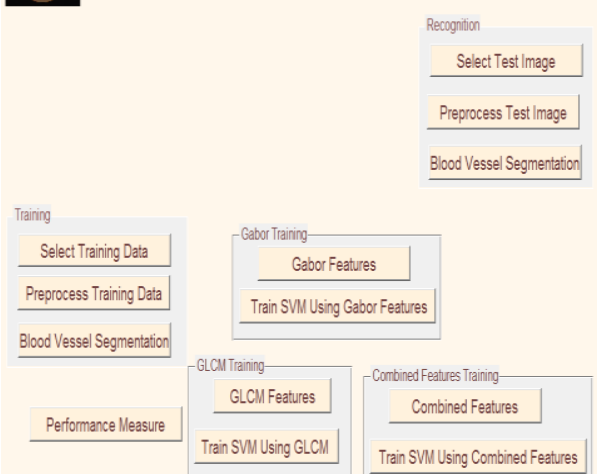

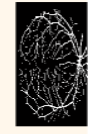

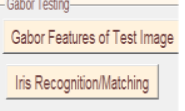

Irs Recognition Matching

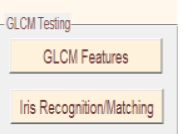

- Contined Featres Testing Combined Features Ins RecognitionMatching

Figure 2 Proposed System of iris recognition by using blood vessel segmentation before the result

Table 1 Result Of Drive Images Dataset

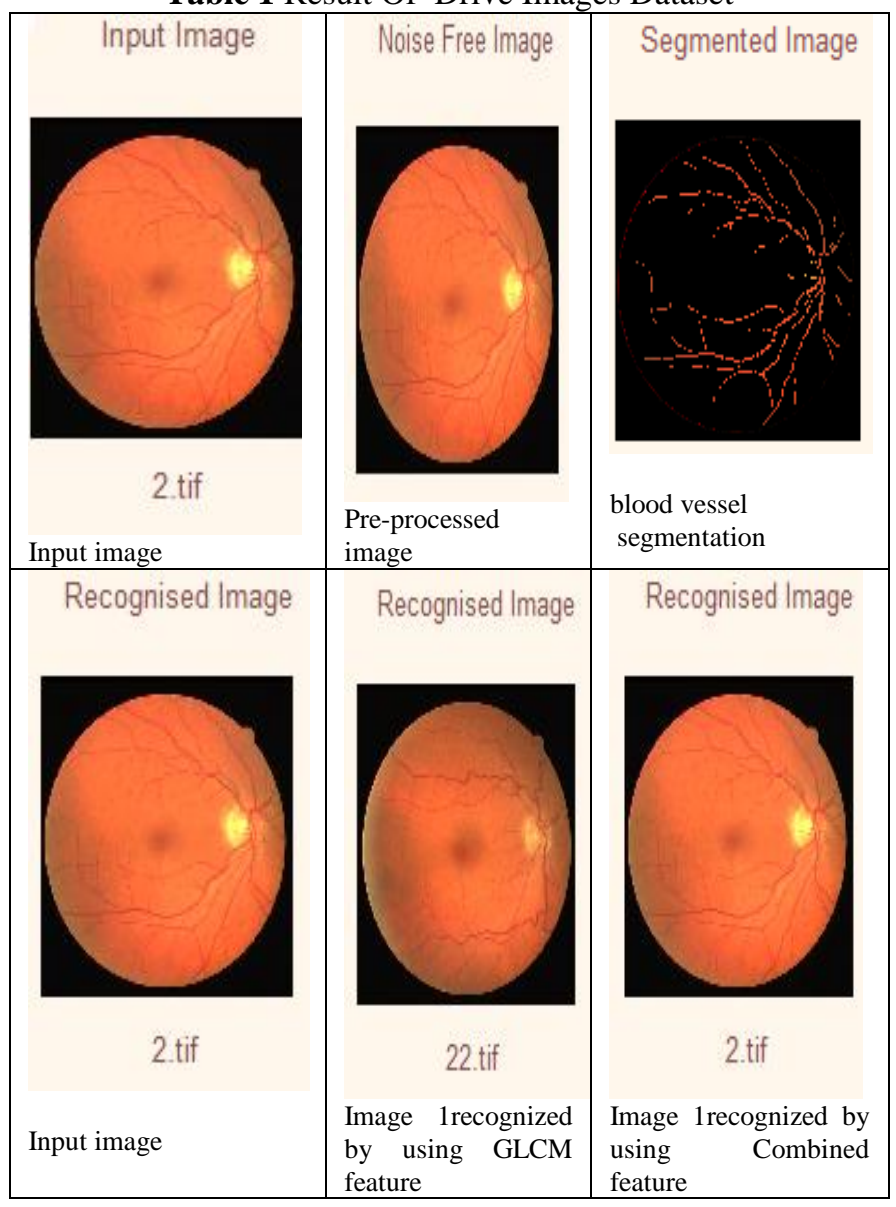


Table 2 percentage of drive dataset images feature

\begin{tabular}{|l|l|l|l|}
\hline No. & Features & Percentage of Correct Classification & Percentage of Incorrect Classification \\
\hline 1 & GLCM Features & 82.50 & 17.5 \\
\hline 2 & Gabor Wavelet Features & 62.50 & 37.5 \\
\hline 3 & Combined Features & 87.50 & 12.5 \\
\hline
\end{tabular}

Graph analysis

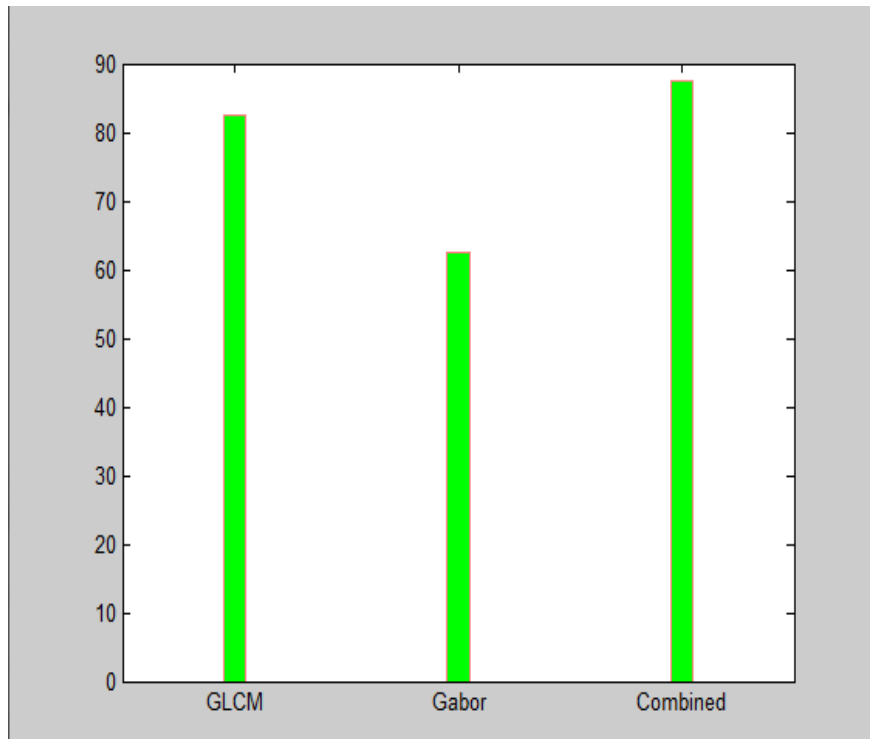

Fig 3 graph analysis of drive dataset

\section{Conclusions:}

Iris recognition process is carried out by using blood vessel segmentation. The system presented an approach for authenticating a person through his own unique retina pattern. Although the number of retina images was used to justify the proposed method were not very high, the results found were very interesting and indicative of the robustness of the method.

The space and time complexity is reasonable, since we found that each retina images contains no more than 100 of intersection points. Iris Recognition is more secured and reliable methodology for authentication preventing direct attacks. Iris recognition technology is durable, quantifiable, recordable and reliable. It thus fulfils the basic tenets of an ideal biometric system. The stored biometric template can be used for a person's whole life as iris patterns are not susceptible to change, remaining stable for long periods of time. Enrolment is required only once in a lifetime, saving both time and money. iris recognition systems are easy to use and create a hassle free security environment. Iris scanners can be used to protect high value locations by denying access to unwarranted visitors. Business and governmental organizations across the board have recognized the benefits of this system and have gone about implementing iris recognition based authentication systems in a big way.

\section{References}

[1]. S. Prabhakar, S. Pankanti, and A. K. Jain, "Biometric recognition: Security and privacy concerns", IEEE Security and Privacy, vol. 1, No. 2, pp. 33-42, 2003.

[2]. Ravi Das, "Retinal recognition Biometric technology in practice", Keesing Journal of Documents \& Identity, vol. 22, pp. 11-14, 2007.

[3]. Charles Boncelet (2005).’Image Noise Models". In Alan C. Bovik. Handbook of Image and Video Processing.

[4]. Priyanka Shrivastava, Uday Pratap Singh, "Noise Removal using First Order Neighborhood Mean Filter", 978-1-4799-30647/14/\$31. 00, 2014 IEEE.

[5]. Fu Bin, Xiong XinyanI, Sun Guozhen, "An efficient Mean Filter Algorithm”,, Proceedings of the 2011 IEEEIICME International Conference on Complex Medical Engineering May 22 - 25, Harbin, China.

[6]. Yi Hong, Sam Kwong, Hanli Wang, "Decision-Based Median Filter Using K-Nearest Noise-Free Pixels", ICASSP 2009, 978-1-42442354-5/09/\$25.00,2009 IEEE.

[7]. B. Sravani, M. V. N ageswara Rao, "Removing of high density salt and pepper noise using Fuzzy Median Filter", 978-1-4799-59587114/\$31.00, 2014 IEEE.

[8]. []. Jacob Benesty, Jingdong Chen, and Yiteng (Arden) Huang, "Study of the Widely Linear Wiener Filter for Noise Reduction", ICASSP 2010, 978-1-4244-4296-6/10/\$25.00, 2010 IEEE.

[9]. SmitTrambadia, Paresh Dholakia, "Design and Analysis of an Image Restoration Using Wiener Filter with a Quality Based Hybrid Algorithms", IEEE Sponsored 2nd International Conference on Electronics and Communication System (ICECS 2015), 978-1- 4788$7225-8 / 15 / \$ 31.00,2015$ IEEE. 
[10]. Peng Xuange,Xiao Ying, Zhu Bing, Liu Yong, "River Sediment image denoising algorithm based on multidirectional 1D wavelet Transform”, 2009 International Conference on Information Management, Innovation Management and Industrial Engineering, 978-07695-3876-1/09 \$25.00, 2009 IEEE DOI 10.1109/ICIII.2009.215

[11]. Hong Yang,Yiding Wang, “An Improved Method of Wavelets Basis Image Denoising Using Besov Norm Regularization”, Fourth International Conference on Image and Graphics, 0-7695-2929-1/07 \$25.00, 2007 IEEE DOI 10.1109/ICIG.2007.143

[12]. Awanish Kumar Shukla,R. L. Verma, Mohd. Sanawer Alam, Vikrant Bhateja, "Directional Order Statistics Filtering for Suppression of Salt and Pepper Noise", 2014 International Conference on Signal Processing and Integrated Networks (SPIN). 978-1-4799-28668/14/\$31.00, 2014 IEEE.

[13]. Bram Cornelis, Marc Moonen, and Jan Wouters, "Performance Analysis of Multichannel Wiener Filter-Based Noise Reduction in Hearing Aids Under Second Order Statistics Estimation Errors", IEEE Transactions On Audio, Speech, And Language Processing, VOL. 19, NO. 5, JULY 2011

[14]. Fraz,P.Remagnino,A.Hoppe,B.Uyyanonvara,A.Rudnicka,C.Owen,Barman, "Blood vessel segmentation methodologies in retinal images - a survey", Computer Methods and Programs in Biomedicine.

[15]. M. J. Dallwitz (1980). A general system for coding taxonomic description [online]. vol. 29. Available:http://delta-intkey.com .

[16]. H. Fu et al., "Machine learning techniques for ontology-based iris classification,"

[17]. X. F. Wang et al., "Recognition of iris images based on shape features using hypersphere classifier," ICIC, vol. 3644/2005, pp. 87-96, 2005.

[18]. A. vyas, B. Talati and S. Naik, "Colour Features Extraction Techniques of Fruits: A Survey", International Journal of Computer Application, Vol 83 - No 15, December 2013. N

[19]. G. Moreda et al. ,"Shape determination of horticultural produce using two- dimensional computer vision - A Review", Journal Food Enginering, Vol 108 - pp. $245-261,2012$.

[20]. G. Moreda et al. ,"Non-destructive technologies for fruit and vegetables size determination - review", Journal of Food Engineering, Vol 92 - pp. 119 - 136, 2009.

[21]. Ming-Kuei Hu, "Visual Pattern Recognition By Moment Invariants" Ire Transactions On Information Theory.

[22]. Krishna Singh, Indra Gupta, Sangeeta Gupta, SVM-BDT PNN and Fourier Moment Technique for classification of iris, International Journal of Signal Processing, Image Processing and Pattern Recognition Vol. 3, No. 4, December, 2010

[23]. Jyotismita Chaki, Ranjan Parekh, "Designing An Automated System For iris Recognition", International Journal Of Advances In Engineering \& Technology, Jan 2012, ISSN: 2231-1963..

[24]. X. Wang, D. Huang, J. Dua, H. Xu, L. Heutte, "Classification of plant iris images with complicated background”,Special Issue on Advanced Intelligent Computing Theory and Methodology in Applied

[25]. R.M. Haralick, Statistical and Structural Approaches to Texture, Proc. IEEE, 67, 1979, pp.786-804.

[26]. R.M. Haralick and L.G. Shapiro, Computer and Robot Vision, Vol. 1, Addison-Wesley, Reading, MA, 1992.

[27]. T.R. Reed and J.M.H. Du Buf, A Review of Recent Texture Segmentation and Feature Extraction Techniques, CVGIP: Image Understanding, 57, 1993, pp. 359-372.

[28]. C.H. Chen, L.F. Pau, and P.S.P. Wang, Handbook of Pattern Recognition and Computer Vision, World Scientific, Singapore, 1993.

[29]. L. Van Gool, P. Dewaele and A. Oosterlinck, Texture Analysis Anno 1983, Comput. Vision, Graphics, Image Process, 29, 1985, pp. 336-357.

[30]. T. Ojala, K. Valkealahti, E. Oja, and M. Pietikainen, Texture Discrimination with Multidimensional Distributions of Signed Graylevel Differences, Pattern Recognition, 34, 2001, pp. 727-739.

[31]. R. W. Conners, M. Trivedi, and C.A. Harlow, Segmentation of a High Resolution Urban Scene using Texture Operators, Computer Vision, Graphics, and Image Processing, 25, 1984, 273-310.

[32]. P. P. Ohanian and R. C. Dubes, Performance Evaluation for Four Classes of Textural Features, Pattern Recognition, 25(8), 1992, 819 833.

[33]. C.C. Gotlieb, and KH.E. Kreyszig, Texture Descriptors based on Co-occurrence Matrices, Computer Vision, Graphics, and Image Processing, 51, 1990, 70-86.

[34]. Y.Q. Chen, M.S. Nixon, and D.W. Thomas, Statistical Geometric Features for Texture Classification, Pattern Recognition, 28 (4), 1995, pp. 537-552.

[35]. P. Brodatz, Textures: A Photographic Album for Artists and Designers, Dover, New York, 1966

[36]. R.M. Haralick, K. Shanmugam and I. Dinstein, Textural Features for Image Classification, IEEE Trans. on Systems, Man, and Cybernetics SMC-3, 1973, pp. 610-621.

[37]. R.F. Walker, Adaptive Multi-scale Texture Analysis with Application to Automated Cytology, Ph.D. Thesis, The University of Queensland, 1997.

[38]. B. Julesz, Visual Pattern Discrimination, IRE Transactions on Information Theory IT-8(2), 84-92, (1962).

[39]. Improving Iris Recognition Performance Using Segmentation, Quality Enhancement, Match Score Fusion, and Indexing By: Mayank Vatsa, Student Member, IEEE, Richa Singh, Student Member, IEEE, and Afzel Noore, Member, IEEE May 2014

[40]. "A Secure Personal Identification System Based on Human Retina" By: Joddat Fatima y, Adeel M. Syed z and M. Usman Akram_International Journal \& Magazine of Engineering, Technology, Management and Research, Volume No: 2 (2015), Issue No: 3 (March). Department of Computer \& Software Engineering Bahria University, Islamabad, Pakistan

[41]. "Personal Identification algorithm based on Retinal Blood Vessels Bifurcation" By: Manjiri B. Patwari Institute of Management Studies \& Information Technology, Vivekananda International Conference on Intelligent Computing Applications May 2014

[42]. Personal Identification System Based on Vascular Pattern of Human Retina” By: Sana Qamber †, Zahra Waheed $\ddagger$ and M. Usman Akram*

[43]. Department of Computer \& Software Engineering Home 2012 Cairo International Biomedical Engineering Conference (CIBEC) Cairo, Egypt, December 20-21, 2012

[44]. Personal Identification based on Human Iris" By: Kezia R Badhiti1, T Sudha2 International Journal of Application or Innovation in Engineering \& Management (IJAIEM) Volume 2, Issue 6, June 2013

[45]. Kecman, V., Learning and Soft Computing, MIT Press, Cambridge, MA. 2001.

[46]. Suykens, J.A.K., Van Gestel, T., De Brabanter, J., De Moor, B., and Vandewalle, J., Least Squares Support Vector Machines, World Scientific, Singapore, 2002

[47]. Scholkopf, B., and Smola, A.J., Learning with Kernels, MIT Press, Cambridge, MA. 2002.

[48]. Cristianini, N., and Shawe-Taylor, J. (2000). An Introduction to Support Vector Machines and Other Kernel-based Learning Methods, First Edition (Cambridge: Cambridge University Press). http://www.support-vector.net/ 